\title{
A propos de perspectives, de détours et d'objectifs
}

\section{Werner Bauer}

Dr méd., président de I'Institut suisse pour la formation médicale postgraduée et continue (ISFM)

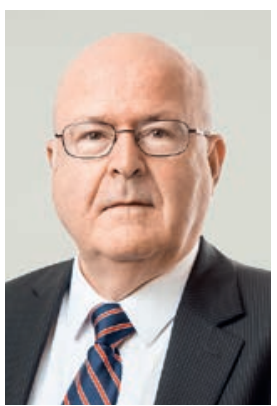

Ce numéro du Bulletin des médecins suisses présente un compte rendu du $5^{\mathrm{e}}$ symposium MedEd de l'Institut suisse pour la formation médicale postgraduée et continue (ISFM). Différents exposés, ateliers et une table ronde ont exploré les perspectives de la formation médicale.

Le choix du terme de formation est délibéré. Il souligne le souhait persistant d'étendre le champ de formation des médecins au-delà du domaine purement technique de la formation prégraduée, postgraduée et continue. C'est aussi pourquoi le catalogue des objectifs d'apprentissage canadien, le CanMEDS, adopté à maintes reprises, définit explicitement l'expert médical ou le promoteur de la santé, mais aussi l'érudit (savant) et le communicateur, parmi les rôles du médecin. Le légendaire William Osler a également insisté sur le fait que «les médecins ont besoin de culture» tout en reconnaissant à la culture générale, large et éclectique (de la littérature aux arts plastiques), une vertu et une aide pour faire face aux exigences professionnelles. La tendance devrait donc aller vers un approfondissement de la formation sans oublier que la réalité du système de santé exige une énergie particulière, pas toujours disponible, pour enseigner suffisamment bien les compétences techniques mais aussi des objectifs plus généraux.

D'autres points du compte rendu méritent d'être relevés, par exemple le "petit échange d'escarmouches avec le directeur de l'Office fédéral de la santé publique» en ouverture de la séance et «l'aimable courrier que l'OFSP envoie occasionnellement à l'ISFM». Cet aimable courrier inclut en fait 45 lettres du Département de l'intérieur qui attestent la reconnaissance des programmes de tous les titres de spécialiste pour les sept prochaines années. Selon la loi, l'octroi des titres de spécialiste sur mandat de la Confédération est conditionné par une accréditation périodique qui, cette année, a certifié la qualité élevée du travail des sociétés de discipline et de l'ISFM après une évaluation approfondie. Pour l'heure, tout va bien et nous sommes ravis de ce courrier plein de reconnaissance. Mais l'OFSP ne se contente pas de cette coopération réjouissante. Il lui arrive occasionnellement de lui préférer des détours pas toujours compréhensibles. Je ne me ré- fère pas ici aux questions tarifaires controversées mais à un état d'esprit qui semble s'imposer au détriment d'une collaboration basée sur le partenariat, sans que ce constat peu flatteur ne se limite à l'OFSP ou à l'Administration fédérale. Si certains services administratifs étaient des organismes vivants, une analyse génomique montrerait certainement deux gènes dominants: le premier, qui déclenche une profonde méfiance à l'égard des activités autonomes du corps médical et le second, qui stimule la synthèse entre de nouvelles réglementations et des mécanismes de contrôle plus rigoureux. C'est ainsi que la saga des lois et ordonnances suit son cours...

Le compte rendu soulève également une question cruciale matérialisée par les observations de l'orateur irlandais selon lequel la formation postgraduée doit entrer dans le XXI ${ }^{\mathrm{e}}$ siècle et ne pas rester figée dans la méthodologie surannée du XIX ${ }^{\mathrm{e}}$. Ce chirurgien a attiré l'attention sur les restrictions du temps de travail et le nombre pas toujours suffisant de patients, qui impactent un entraînement technique efficace. $\mathrm{Si}$, au premier abord, cela peut sembler être une lapalissade, il est là aussi question de détours qui ne permettent pas de cibler avec détermination les objectifs. Il est indispensable de développer des méthodes plus efficaces (simulation, formation mixte) pour transmettre et utiliser les compétences, de réduire enfin la charge administrative et d'harmoniser les liens entre prestation, besoin de formation postgraduée et temps de travail.

La dernière remarque concerne la communication, dont le rôle primordial ne cesse d'être souligné; et pourtant, de nombreux problèmes sur le lieu de travail ou dans les relations entre les patients et les médecins découlent d'un manque de communication ou d'une communication inappropriée ou insensible. L'orateur de la Mayo Clinic a rappelé que l'art et la manière de transmettre un message sont tout aussi importants que le message véhiculé, qu'il s'agisse d'une présentation, d'une discussion dans le quotidien de la formation ou d'un entretien avec un patient. L'impact de la communication est trop souvent négligé.

Conclusion: les perspectives sont bonnes mais elles ont besoin d'un terrain fertile pour se réaliser. 